Patil, SB; Vögtli, M; Webb, B; Mazza, G; Pinzani, M; Soh, YA; McKendry, RA; (2015)

Decoupling competing surface binding kinetics and reconfiguration of receptor footprint for ultrasensitive stress assays. Nat Nanotechnol 10.1038/nnano.2015.174. Downloaded from

UCL Discovery: http://discovery.ucl.ac.uk/1470016

\title{
ARTICLE
}

\section{Decoupling competing surface binding kinetics and reconfiguration of receptor footprint for ultrasensitive stress assays}

Samadhan B. Patil ${ }^{1,2 \dagger}$, Manuel Vögtli' ${ }^{1 \dagger}$, Ben Webb ${ }^{1,3}$, Giuseppe Mazza ${ }^{4}$, Massimo Pinzani ${ }^{4}$, Yeong-Ah Soh ${ }^{2}$, Rachel A. McKendry ${ }^{1}$ and Joseph W. Ndieyira ${ }^{1,5 t *}$

1. London Centre for Nanotechnology and Departments of Medicine and Physics University College London

17-19 Gordon Street

London, WC1H OAH

2. Department of Materials, Imperial College London, London SW7 2AZ, UK.

3. University College London, Division of Infection and Immunity, UK.

4. UCL Institute for Liver and Digestive Health, Royal Free Hospital London, NW3 2QG

5. Jomo Kenyatta University of Agriculture and Technology

Department of Chemistry

Po Box 62000

Nairobi, Kenya

${ }^{\dagger}$ Authors contributed to experiments

*Corresponding author: Joseph Ndieyira Email: (j.ndieyira@ucl.ac.uk)

\begin{abstract}
Cantilever arrays have been used for monitoring biochemical interactions and their associated stress. However, it is often necessary to passivate the underside of the cantilever to prevent unwanted ligand adsorption and this process requires tedious optimization. Here we show a way to immobilize membrane receptors on nanomechanical cantilevers such that they can function without passivating the underlying surface. Using equilibrium theory, we quantitatively describe the mechanical response of vancomycin, human immunodeficiency virus type 1 antigens and coagulation factor VIII captured on the cantilever in the presence of competing stresses from both the top and bottom cantilever surfaces. We show that the area per each receptor molecule on the cantilever surface influences ligand-receptor binding complexation and plays an important role on stress. Our results offer a new way to sense biomolecules and, will aid in the creation of ultrasensitive biosensors.
\end{abstract}


Chemo-mechanical signaling - whereby mechanical activities of ligand molecules such as proteins are coupled to chemical events - is important in the regulation of physiological processes in biological systems ${ }^{1-3}$. Ligand binding to membrane receptors forms complex structures that activate signaling pathways. This transduces a distinct output signal that effects functional changes in living systems. Furthermore, mechanical interactions governing transport and antibody affinity at the molecular level also determine cellular adhesion and motility ${ }^{4}$. The interplay between chemistry and mechanics controls how cells communicate with each other and their environment ${ }^{5}$.

Understanding cellular mechanical signal transduction can help us design sensors that have better ligand detection specificity and sensitivity. We use cantilever arrays to redefine the limit of molecular recognition with direct mechanical sensing because they are well suited to monitoring biochemical interactions ${ }^{6-8}$ and the associated stresses $^{9,10}$. Cantilevers can sense the replication of bacterial cells ${ }^{11}$, bacterial vitality $^{12}$, the binding kinetics of antibiotics ${ }^{13,14}$, perform nanoscale mapping and the functional analysis of individual adhesins on living bacteria ${ }^{15}$, and detect interferon- $\alpha$ induced I-8U gene expression in total human RNA, a potential marker for melanoma progression and viral infections ${ }^{16}$. Neighbouring cantilevers in a given chip, when coated with moieties recognising different ligands of interest, can enable multiplexed, simultaneous and label-free detection of biomolecules. Cantilevers are made up of micro-meter-thin silicon beams whose mechanical movements and natural frequencies are exquisitely sensitive to physical or chemical changes. Cognate ligand recognition leads to two classes of perturbation within cantilevers: (1) downshifts in the resonance frequency of each vibrational mode (dynamic mode) ${ }^{17-19}$ and (2) as a result of stress generated from such interactions, cantilever bending moments (static mode $)^{13,14,20}$. In our previous studies ${ }^{13,14}$ we investigated the roles of geometry and chemistry for the performance of cantilever biosensors and quantified free antibiotic concentrations in blood serum to define the effectiveness of drug dosages required for different individuals and to reduce the potential toxic side effects. However, it remains unclear how competing binding interactions at the top ( $\mathrm{Au}$ ) and bottom (Si) surfaces of the cantilever can be optimised to control signal amplification as well as specificity to create an ultrasensitive biodetection system.

Here, we draw inspiration from chemo-mechanically induced signals found in biological systems to develop a diagnostic tool that exploits biomechanics of ligandreceptor interactions to characterise, in real time, the activities of antibiotics, viral antigens and clotting factors for bleeding related disorders. We formulate a mathematical model to decouple the binding kinetics at $\mathrm{Au}$ and Si surfaces of the cantilever in parallel and under identical conditions (Fig. 1). As depicted in Fig. 1a-c, we show a way to immobilise membrane receptors on nanomechanical cantilevers so that they can be operated without the need to passivate the underlying surface. In Fig. 1d, we show that the passivation of the cantilever underside is consistent with using a single surface for sensing measurements. We propose the hypothesis that molecular footprint of membrane receptors (area per each receptor molecule) on the cantilever surface determines the driving force necessary to cause complexation 
between an incoming ligand and receptor. We test this hypothesis using a panel of biologically relevant molecules.

In biospecific sensing measurements it is often essential to passivate the underside of the cantilever to prevent unwanted ligand adsorption ${ }^{21,22}$. However, this requires extensive optimization. We therefore explored whether cantilevers can function without underside passivation. As a proof of concept we functionalized both surfaces of the cantilever (Fig. 1a-c) with a self-assembled monolayer (SAM) of vancomycin (Van) susceptible receptor (VSR $\sim 0.6 \mathrm{kDa}$ ) analogues of the bacterial cell wall precursors that present uncrosslinked peptide motifs terminating in thesequence lysine-D-alanine-D-alanine ${ }^{14,23,24}$. To eliminate the artefacts that produce nonspecific signals we performed differential measurements in which we subtracted reference polyethylene glycol (PEG)-coated cantilever bending signals from the receptor signals. This functionalization was performed without pre-adsorbing a resistive protein monolayer of bovine serum albumin (BSA) ${ }^{22}$ or PEG-silane ${ }^{21}$, which are known to block non-specific interactions (Supplementary Case I). Van was used as a reporter molecule because it reacts specifically with VSR to generate stress, which leads to cantilever bending deflections ${ }^{13,14,20}$. Moreover, Van-the Food and Drug Administration (FDA)-approved drug of last resort in the clinical treatment of bacterial infections caused by methicillin-resistant Staphylococcus aureus (MRSA), Clostridium difficile infections (CDIs) and the recently identified multidrug-resistant clones of $\mathrm{MRSA}^{25}$-is highly desirable as a model drug to analyse in detail the mechanisms of action of antibiotics.

To probe stresses due to antibiotic binding to different sensing surfaces, we injected Van to unpassivated cantilvers functionalised with VSR. The outcome after addition of $250 \mu \mathrm{M}$ Van is summarised in Figure 2a. The bending response (as shown in Figure 2a) is caused by the interactions of Van molecules at the surface, giving rise to the formation of Van-VSR complex that induces a local strain in the cantilever as well as carry an electrostatic positive charge ${ }^{26}$ under physiologically-relevant environment. The electrostatic repulsive and steric interactions between Van-VSR complexes create a compressive stress at $\mathrm{Au}$, causing cantilevers to bend downwards. The reference PEG coated cantilevers, as expected, showed no bending response against Van. The mechanical response generated from Van-VSR complex interactions increased with increasing VSR concentration but decreased at high concentration of $1000 \mu \mathrm{M}$.

The effect of VSR concentration on signal amplification is summarised in Fig. 2b. We find that the stress response against VSR concentration is categorised by two regimes (I and II). Regime I represents the initial stages of self-assembly of molecules and is characterised by a sharp rise of compressive stress of up to 52 $\mathrm{mNm}^{-1}$ when the concentration of VSR in solution is $50 \mu \mathrm{M}$. This is approximately two times more sensitive than previous measurements ${ }^{14}$ where the net stress was 33 $\mathrm{mNm}^{-1}$. However, as the VSR concentration is increased beyond $50 \mu \mathrm{M}$, termed regime II, we find a significant decrease of stress signals down to $\sigma_{\max } \sim 5 \mathrm{mNm}^{-1}$. In contrast, we observed zero differential stress for the reference PEG coated cantilevers. As a further measurement control, we used uncoated $\mathrm{Au}$ and $\mathrm{Si}$ surfaces. The undetectable mechanical response in the presence of Van is an 
additional verification that the observed deflection signal is caused by the interactions of Van with VSR. In general, non-monotonic stress signal changes observed in Fig. $2 \mathrm{~b}$ is not surprising given that the reported SAM formation on $\mathrm{Si}^{27-29}$ can give rise to negative contributions to the net cantilever stress signal. To explain the origin of mechanotransduction in case of $\mathrm{Si}$ surface, we assume that individual SAM molecules are oriented with an angle of tilt away from the surface normal ${ }^{30}$. An orientation where a SAM molecule is in a 'lying-down' conformation is disordered and is unlikely to yield significant stress.

\section{Ligand sensing based on surface plasmon resonance (SPR)}

With stress signal reduction at Au surface occurring at high VSR concentrations (Fig $2 b)$ our next objective was to confirm that these changes are caused by the opposing Si reactions. We therefore employed a commercially available SPR method (see Supplementary Fig. S1 and Supplementary Section 'case II'), where the detection of biochemicals is at a single planar metal surface ${ }^{31,32}$. The SPR detection is based on monitoring the changes in dielectric properties caused by ligand adsorption. Accordingly, we performed a series of binding analysis of VSR, for which [Van] was kept constant at $250 \mu \mathrm{M}$ to match the experimental conditions for the cantilever based measurements. In Fig. 2c, we show the differential SPR signal response increases with increasing VSR concentration. Furthermore, the SPR response displays an s-shaped curve undergoing a steep rise before reaching a plateau, when the receptor concentration is increased beyond $10 \mu \mathrm{M}$ (Fig. 2d). The SPR analysis of signal response against VSR concentration shown in Fig. $2 \mathrm{~d}$ remains constant even when [VSR] is extended to $1000 \mu \mathrm{M}$ and, when compared with direct mechanical quantitation (Fig. 2b), demonstrates that the reduction of stress signals at higher VSR concentration is linked to underside Si reactions. Our measurements provide the first demonstration that direct functionalisation of cantilevers, without underside passivation (Fig. 1d), can be achieved by the effective tuning of receptor concentrations in solution described herein. Previous measurements using cantilevers have focused on one side only ${ }^{21,22}$, but it is essential to understand how the underlying Si surface affects the overall mechanical response.

\section{Modelling of surface functionalisation for biosensors}

We devised a model (model I), illustrated in Fig. 1a, to represent the simultaneous interactions at the Au and Si surfaces, where the surface stress is defined by ligandreceptor complex interactions. The net change in stress is expressed as

$$
\Delta \sigma_{e q}=\sigma_{\max }(A u)\left(\frac{[\text { Ligand }]^{n}}{K_{d}^{n}(\text { Au })+[\text { Ligand }]^{n}}\right)+\sigma_{\max }(S i)\left(\frac{[\text { Ligand }]^{m}}{K_{d}^{m}(S i)+[\text { Ligand }]^{m}}\right)
$$

where the first term in Eq. (1) quantifies stress changes at Au surface and the second term is at Si surface. For the associated stress to cause an effective cantilever downward bending (compressive) with an inclusion of the bound complex, the Au top must expand, meaning the underlying Si surface undergoes contraction (tensile). The constants $\sigma_{\max }(\mathrm{Au})$ and $\sigma_{\max }(\mathrm{Si})$ are the maximum stresses when all accessible binding sites on the surfaces are fully occupied. $K_{d}(\mathrm{Au})$ and $K_{d}(\mathrm{Si})$ are the 
equilibrium dissociation constants for Au and Si surfaces, whereas $n$ and $m$ are the stoichiometric coefficients of the reactions.

Assuming $n=m=1$, the global fit of Eq. (1) to the stress data is shown in Figure $2 \mathrm{~b}$ using four fitting parameters. The calculated $K_{d}(\mathrm{Au})$ and $K_{d}(\mathrm{Si})$ for VSR on $\mathrm{Au}$ and Si surfaces are $0.6 \pm 0.2 \mu \mathrm{M}$ and $200 \pm 10 \mu \mathrm{M}$ respectively. The maximum stress signals $\sigma_{\max }$ generated at Au was $52 \pm 3 \mathrm{mNm}^{-1}$ and $42 \pm 3 \mathrm{mNm}^{-1}$ for Si. We find that $K_{d}(\mathrm{Si})$ is large because $\mathrm{Si}$ underside, in contrast to $\mathrm{Au}$, tends to easily form oxides ${ }^{33}$ but the formation of Si-S compounds requires desorption of oxide contaminants and other impurities ${ }^{30}$, therefore hindering the binding kinetics of SAM. If we overlook the contributions from the underlying $\mathrm{Si}$ reactions, the actions of cantilever (Au surface) matches the SPR response. Thus, if $\sigma_{\max }(\mathrm{Si})$ is set to zero, model (1) reduces to model (II), and the net change in SPR signal is expressed as

$$
R U_{e q}=R U_{\max }(A u)\left(\frac{[\text { ligand }]^{n}}{K_{d}^{n}(A u)+[\text { ligand }]^{n}}\right)
$$

where $R U_{\text {eq }}$ is the equilibrium response units, and $R U_{\max }(\mathrm{Au})$ is the maximum response units when all active sites are occupied. Accordingly, we fitted Eq. (2) to the SPR data (Fig. 2d), with 3 parameters. The calculated $K_{d}(\mathrm{Au})$ for VSR was $0.5 \pm 0.1$ $\mu \mathrm{M}$ while the stoichiometric coefficient and maximum response units are 1 and 2100 \pm 100 respectively. These findings demonstrate that cantilevers using equation (1) give the same dissociation constants of $K_{d}(\mathrm{Au})$ of $0.6 \pm 0.2 \mu \mathrm{M}$ for VSR as the SPR method, matching the reported high affinity of SAMs for the surfaces of noble metals $^{30}$. The consistency between SPR and mechanical assays is not surprising, even though there is no bending response in SPR sensing in contrast to cantilevers where there is a bending response as result of stress. It is therefore possible that the net energy of formation involved in ligand binding derived from hydrogen bonding, electrostatic interactions, hydrophobic, and Van-der-Waals forces is unlikely to be influenced by whether there is a bending response or not. By cantilever bending, the distance between receptor-head-groups would be altered by a small fraction, but won't have a significant impact on the binding efficacy.

We next explored the impact of non-passivation on direct mechanical assays. The stress response was monitored after injecting Van at concentrations of 1, 10 and 250 $\mu \mathrm{M}$ against VSR concentrations initially fixed at $50 \mu \mathrm{M}$ (Fig. 3a), where full surface coverage was established. We found that as [Van] increases, the corresponding stress response increases accordingly. Moreover, to examine the reproducibility of bending signals, we performed 10 measurements of Van at each receptor concentration using four different chips, making a total of 6500 measurements with the results summarised in Figure $3 \mathrm{~b}$. The calculated $K_{d}(\mathrm{Au})$ for Van using equations (1) and (2) was $0.6 \pm 0.1 \mu \mathrm{M}$ and $0.52 \pm 0.13 \mu \mathrm{M}$ respectively, in good agreement with the previous measurements ${ }^{13}$. However, we found that $K_{d}(\mathrm{Au})$ increases by more than an order of magnitude when VSR concentration is below $1 \mu \mathrm{M}$ or greater than $1000 \mu \mathrm{M}$. To demonstrate the accuracy of these findings, we make comparisons with SPR methodology (Fig. 3c, d). Figure 3e shows a summary of $K_{d}(\mathrm{Au})$ at different VSR concentrations. The $K_{d}(\mathrm{Au})$ obtained using SPR method is in good 
agreement with the cantilever technique and increased to $27.9 \pm 13.2 \mu \mathrm{M}$ when the VSR limit was decreased below $1 \mu \mathrm{M}$. To account for the increase in $K_{d}(\mathrm{Au})$ at low VSR concentration, we consider that the net cantilever stress signal contribution from both surfaces is small because a complete monolayer is not formed at such concentration ${ }^{30}$. We believe that high dissociation constant is a measurement artefact due to the fact that stress is not only determined by the chemical binding efficiency but also by a geometric factor ${ }^{14}$ that is smaller than one at coverages below a full monolayer. Correspondingly, the high dissociation constant obtained by SPR measurements at [VSR] concentrations below $1 \mu \mathrm{M}$ is also explained as an artefact of the measurement of the index of refraction. Conversely, when [VSR] concentration is greater than $1000 \mu \mathrm{M}$, a large contribution from Si reactions comparable to that from the Au top surface results in the reduction of the net stress signal (Fig. 2b). Subsequently, the cantilever measurements yield a large $K_{d}(\mathrm{Au})$, which is an artefact because the stress contribution from the $\mathrm{Si}$ reactions counteracts that of the $\mathrm{Au}$ surface (equation (2)). Thus, when either the VSR concentration is too low or higher than $1,000 \mu \mathrm{M}$, the extracted $K_{d}(\mathrm{Au})$ values are artefacts. Consequently, a sufficient equilibrium net stress signal,

${ }_{e q}$ or equilibrium SPR response units are required for accurate binding analysis.

\section{Effect of receptor surface footprint on the ligand binding}

Although it is understood that receptor-ligand interactions in solution are linked to stress generation ${ }^{13,14}$, it is unclear how the surface footprint correlates with the concentration of receptors in solution. The results showing that surface coverage is a function of the receptor concentration in solution is summarised in the Supplementary Tables S1 and S2 and Fig. 3f. To study the impact of receptor spacing on stress generation efficiency, we incorporated a receptor molecule with a second SAMforming molecule PEG on the Au surface ${ }^{34,35}$ without underside passivation (Fig.1ac). We chose PEG because it resists unwanted adsorptions of ligands ${ }^{34,35}$ by acting as a protein "repellent". Moreover, it acts as a 'spacer' in varying the distribution of receptors on the surface whilst simultaneously controlling the accessibility of ligands. Fig. $4 \mathrm{a}$ shows the outcome when cantilevers were exposed to a constant antibiotic concentration at $250 \mu \mathrm{M}$ Van after a defined ratio of VSR/PEG, where the total receptor concentration was fixed at $1 \mu \mathrm{M}$ to minimise the negative impact of $\mathrm{Si}$ reactions. Here, we find an intriguing behaviour in mechanical response. For the sparsely distributed receptor of $\sim 30 \%$, the cantilever deflection signal is negligible. However, when receptor concentration is increased to $100 \%$, the surface packing density is maximised and yields the highest stress (Fig. 4a). These actions show that the number of ligand-receptor interactions increases with coverage, but there is a threshold in the surface footprint required to generate a mechanical response, in good a greement with our previous studies ${ }^{14}$.

To examine the impact of coverage on signal amplification and to exclude any possibilities of the contributions from $\mathrm{Si}$ reactions, the cantilever underside was passivated (see Supplementary Section 'case III' and Fig. 4b). To provide insight into the dependence of stress generation on molecular size, we tested the N-terminal fragment $(\mathrm{VHH})$ of llama single chain antibodies ${ }^{36}$ which have a molecular weight of about $15 \mathrm{kD}$, some $25 \mathrm{x}$ larger than VSR but only $10 \%$ of a conventional 
immunoglobulin. $\mathrm{VHH}$ are stable over a broad temperature range $\left(-80^{\circ} \mathrm{C}\right.$ to $\left.80^{\circ} \mathrm{C}\right)$ and are inexpensive to manufacture with excellent expression yields from bacteriophage libraries ${ }^{37}$. We chose $\mathrm{VHH}$ raised against the human immunodeficiency virus (HIV-1) trimeric envelope glycoprotein (gp140) as previously described ${ }^{38-41}$. One $\mathrm{VHH}$, termed 2B4F, was chosen because of its high specificity and sensitivity of binding to the gp140 by SPR ${ }^{41}$. Figure 4c shows the outcome after exposure to recombinant antigens derived from HIV-1 subtype $A^{38}$ (gp140UG37, $140 \mathrm{kDa}$ ) fixed at $50 \mu \mathrm{M}$ against defined percentage ratio of receptors at total solution concentration of $2 \mathrm{mM}$. The observed noise was probably caused by the scattering of the laser light by the proteins. The response signal was not detectable at $20 \%$ relative concentration, but increased as the concentration was increased between 80 to $90 \%$. Surprisingly, $100 \%$ receptor concentration in solution was found to yield insignificant stress signals. Generally, our findings reveal that the efficiency of stress generation for proteins is strongly dependent on the surface molecular footprint. In contrast, for small molecules such as Van $(\sim 1.4 \mathrm{kDa})$, the stress is maximised when the receptor packing densities is highest (Fig. $4 \mathrm{a}, \mathrm{d}$ and Supplementary Table S2).

\section{Sensing clinically important large molecules}

The ability of $\mathrm{VHH}$ antibodies to detect antigens from different classes of HIV-1 isolates was further tested by using gp140CN54 glycoprotein ${ }^{38}(\sim 140 \mathrm{kDa})$, an isolate from $B / C$ subtype. The specificity of protein detection was confirmed by using a nonspecific VHH, LAB5 and PEG as references and cantilever underside passivation. Figure $5 \mathrm{a}$ shows the outcome with the limit of detection down to $500 \mathrm{fM}$, a marked increase in sensitivity compared to other studies which have reported detection limits by cantilevers of $500 \mathrm{pM}^{6}$. In comparison to the conventional enzymelinked immunosorbent assays (ELISA), which has reported $\mathrm{nM}$ to $\mathrm{pM}^{42}$ detection limits, the cantilever detection sensitivity is superior.

To quantify $K_{d}(\mathrm{Au})$ of gp140CN54, we averaged measurements over four separate cantilever chips (Fig. 5b). The outcome of the fit of Eq. (2) superposed onto differential stress signals reveals $K_{d}(\mathrm{Au})$ of $6.2 \pm 1.2 \mathrm{pM}$ and $3 \pm 0.1 \mathrm{mNm}^{-1}$ maximum stress signal. To investigate whether sensitivity is determined by the underlying surface chemistry, we measured the binding of gp140CN54, against a covalently attached carboxymethylated dextran in the SPR method (Fig. 5c), revealing a detection sensitivity of $10 \mathrm{nM}$ and $K_{d}(\mathrm{Au})$ of $3.1 \pm 1.4 \mathrm{pM}$. The impact of surface chemistry was further assessed by replicating the cantilever chemistry on unmodified Au-coated SPR sensor chips (see Supplementary Section 'case IV') with $50 \mathrm{nM}$ detection sensitivity (Fig. $5 \mathrm{~d}$ ), which is five orders of magnitude less sensitive than cantilevers. The extent of biochemical detection sensitivity is therefore governed only by the technique itself. The enhanced cantilever sensitivity confirms our hypothesis that sensitive detection is strongly linked to a ligand's ability to polymerize $^{13}$ such as HIV-1 glycoprotein which forms trimeric complexes ${ }^{43}$.

Finally, we assessed whether ligand molecular weight has any impact on the magnitude and direction of cantilever displacements. This was achieved by utilising coagulation Factor (VIII) whose molecular weight $\sim 280 \mathrm{kDa}$, is exactly twice the size of gp140CN54. Figure 6a shows the detection experiment of Factor (VIII) and control experiment in distilled water. The analysis as shown in Fig. 6b, using Eq. (2) reveals 
$K_{d}(\mathrm{Au})$ of $8.8 \pm 4 \mathrm{IUml}^{-1}$ and $\sigma_{\max }(\mathrm{Au})$ of $30.6 \pm 2.8 \mathrm{mNm}^{-1}$. The compressive stress signature we observed for Factor (VIII) could be attributed to the steric crowding and, for the same reasons, we intuitively expected to observe a compressive surface stress for HIV-1 recombinant proteins. Surprisingly, all HIV proteins we investigated caused a tensile surface stress. Here, we speculate that the observed tensile stress is probably caused by Van-der-Waals forces and hydrogen bond formation.

\section{Conclusions}

Our comprehensive experiments show that cantilever arrays have the sensitivity to quantify competing binding interactions at $\mathrm{Au}$ (top) and $\mathrm{Si}$ (bottom) surfaces in parallel. Thus, the surface reaction kinetics are decoupled and by tuning the receptor concentrations, we can minimise the impact of the underlying $\mathrm{Si}$ reactions. The approach provides a new framework for understanding and eventually engineering mechanical responses to biochemical interactions without the need for tedious $\mathrm{Si}$ underside passivations. The findings will aid rational design of novel devices and surface chemistries to improve sensitivity of bioassays in targeting ultra-low concentrations of disease biomarkers. Moreover, our assays establish cantilevers as a host for ultrasenstive and biospecific assays, where the sensitivity to different analytes resides in the underlying surface chemistries. We find that while there is a threshold of receptor density required to generate stress, in some systems, a higher surface coverage does not necessarily lead to enhanced signals and to the contrary can result into undetectable mechanical response. Nanomechancal cantilever sensing is measured in minutes, and raw materials for its manufacture is based on low cost silicon, making this technology an ideal candidate to be used for the development of a Point-of-Care (PoC) diagnostic device that could be paired with customised drug delivery for haemophilia, anti-HIV and antibacterial therapies.

\section{Additional Information}

Supplementary information accompanies this paper at www.nature.com/naturenanotechnology. Reprints and permission information is available online at http://npg.nature.com/reprints and permissions/. Correspondence and requests for materials should be addressed to JWN.

\section{REFERENCES}

(1) Son, K., Guasto, J. S. \& Stocker, R. Bacteria can exploit a flagellar buckling instability to change direction. Nature Physics 9, 494-498 (2013).

(2) Lele, P. P., Hosu, B. G. \& Berg, H. C. Dynamics of mechanosensing in the bacterial flagellar motor. Proc. Natl. Acad. Sci. USA, 110, 11839-11844 (2013).

(3) Gebhardt, J. C. \& Rief, M. Biochemistry force signaling in biology. Science 324, 1278-1280 (2009).

(4) Arlett, J. L., Myers, E. B. \& Roukes, M. L. Comparative advantages of mechanical biosensors. Nature Nanotech. 6, 203-215 (2011).

(5) Müller, D. J. \& Dufrêne, Y. F. Atomic force microscopy as a multifunctional molecular toolbox in nanobiotechnology. Nature Nanotech. 3, 261-269 (2008). 
(6) Huber, F., Lang, H. P., Backmann, N., Rimoldi, D. \& Gerber, C. Direct detection of a BRAF mutation in total RNA from melanoma cells using cantilever arrays. Nature Nanotech. 8, 125-129 (2013).

(7) McKendry, R. A. et al. Multiple label-free biodetection and quantitative DNAbinding assays on a nanomechanical cantilever array. Proc. Natl. Acad. Sci. USA, 99, 9783-9788 (2002).

(8) Wu, G. H. et al. Bioassay of prostate-specific antigen (PSA) using microcantilevers. Nature Biotechnol. 19, 856-860 (2001).

(9) Fritz, J. et al. Translating biomolecular recognition into nanomechanics. Science 288, 316-318 (2000).

(10) Berger, R. et al. Surface stress in the self-assembly of alkanethiols on gold. Science. 276, 2021-2024 (1997).

(11) Gfeller, K. Y., Nugaeva, N. \& Hegner, M. Micromechanical oscillators as rapid biosensor for the detection of active growth of Escherichia coli. Biosensors and Bioelectronics, 21, 528-533 (2005).

(12) Longo, G. et al. Rapid detection of bacterial resistance to antibiotics using AFM cantilevers as nanomechanical sensors. Nature Nanotech. 8, 522-526 (2013).

(13) Ndieyira, J. W. et al. Surface-stress sensors for rapid and ultrasensitive detection of active free drugs in human serum. Nature Nanotech. 9, 225-232 (2014).

(14) Ndieyira, J. W. et al. Nanomechanical detection of antibiotic-mucopeptide binding in a model for superbug drug resistance. Nature Nanotech. 3, 691696 (2008).

(15) Dupres, V. et al. Nanoscale mapping and functional analysis of individual adhesins on living bacteria. Nature Meth. 2, 515-520 (2001).

(16) Zhang, J. et al. Rapid and label-free nanomechanical detection of biomarker transcripts in human RNA. Nature Nanotech. 1, 214-220 (2006).

(17) Kosaka, P. M. et al. Detection of cancer biomarkers in serum using a hybrid mechanical and optoplasmonic nanosensor. Nature Nanotech. 9, 1047-1053 (2014).

(18) Boisen, A., Dohn, S., Keller, S. S., Schmid, S. \& Tenje, M. Cantilever-like micromechanical sensors. Rep. Prog. Phys. 74, 036101-036131 (2011).

(19) Burg, T. P. et al. Weighing of biomolecules, single cells and single nanoparticles in fluid. Nature 446, 1066-1069 (2007).

(20) Shu, W. et al. DNA molecular motor driven micromechanical cantilever arrays. J. Am. Chem. Soc. 127, 17054-17060 (2005).

(21) Backmann, N. et al. A label-free immunosensor array using single-chain antibody fragments. Proc. Natl. Acad. Sci. USA 102, 14587-14592 (2005).

(22) Mukhopadhyay, R. et al. Cantilever sensor for nanomechanical detection of specific protein conformations. Nano Lett., 5, 2385-2388 (2005).

(23) Nieto, M. \& Perkins, H. R. Modifications of acyl-d-alanyl-d-alanine terminus affecting complex-formation with vancomycin. Biochem. J. 123, 773-787 (1971).

(24) Williams, D. H., Maguire, A. J., Tsuzuki, W. \& Westwell, M. S. An analysis of the origins of a cooperative binding energy of dimerisation. Science 280, 711714 (1998). 
(25) Sieradzki, K. et al. The development of vancomycin resistance in a patient with methicillin-resistant Staphylococcus aureus infection. The New England Journal of Medicine 340, 517-523 (1999).

(26) Barna, J. C. \& Williams, D. H. The structure and mode of action of glycopeptides antibiotics of the vancomycin group. Annu. Rev. Microbiol. 38, 339-357 (1984).

(27) West, R. Inorganic chemistry: Two-armed silicon. Nature 485, 49-50 (2012).

(28) Hamers, R. J. Formation and characterization of organic monolayers on semiconductor surfaces. Annu. Rev. Anal. Chem. 1, 707-736 (2008).

(29) Haas, A. The chemistry of silicon-sulfur compounds. Angew. Chem. Internat. Edit. 4, 1014-1023 (1965).

(30) Love, J. C. et al. Self-assembled monolayers of thiolates on metals as a form of nanotechnology. Chem. Rev. 289, 1103-1169 (2005).

(31) Kolomenskii, A. A., Gershon, P. D. \& Schuessler, H. A. Sensitivity and detection limit of concentration and adsorption measurements by laserinduced surface-plasmon resonance. Applied Optics. 36, 6539-6547 (1997).

(32) Stenberg, E., Persson, B., Roos, H. \& Urbaniczky, C. Quantitative determination of surface concentration of protein surface plasmon resonance using radiolabeled proteins. J. Colloid and interface Science 143, 513-526 (1991).

(33) Morita, M., Ohmi, T., Hasegawa, E., Kawakami, M. \& Ohwada, M. Growth of native oxide on a silicon surface. J. Appl. Phys. 68, 1272-1281 (1990).

(34) Prime, K. L. Whitesides, G. M. Self-assembled organic monolayers - model systems for studying adsorption of proteins at surfaces. Science 252, 11641167 (1991).

(35) Prime, K .L. \& Whitesides, G. M. Adsorption of proteins onto surfaces containing end-attached oligo(ethylene oxide) - a model system using selfassembled monolayers. J. Am. Chem. Soc. 115, 10714-10721 (1993).

(36) Hamers-Casterman, C. et al. Naturally occurring antibodies devoid of light chains. Nature 363, 446-448 (1993).

(37) Hultberg, A. et al. Lactobacilli expressing llama VHH fragments neutralise Lactococcus phages. BMC Biotechnology 84, 1-7 (2007).

(38) Strokappe, N. et al. Llama antibodies recognizing various epitopes of the CD4bs neutralize a broad range of HIV-1 subtypes A, B, and C. PLoS One, 7(3): e33298, 1-11 (2012).

(39) McCoy, L. E. et al. Potent and broad neutralization of HIV-1 by a llama antibody elicited by immunization. J. Exp. Med, 209, 1091-1103 (2012).

(40) McCoy, L. E. et al. Molecular evolution of broadly neutralizing Ilama antibodies to the CD4-binding site of HIV-1. PLoS Pathog, 10(12): e1004552, 1-18 (2014).

(41) McCoy, L. E. et al. Broadly neutralizing VHH against HIV-1. Patent application WO 2013036130 A1 (EP2753644A1, US201501158934).

(42) Zhou, F. et al. Sensitive sandwich ELISA based on a gold nanoparticle layer for cancer detection. Analyst 137, 1779-1784 (2012). 
(43) Liu, J., Bartesaghi, A., Borgnia, M. J., Sapiro, G. \& Subramaniam, S. Molecular architecture of native HIV-1 gp120 trimers. Nature 455, 109-113 (2008). 


\section{ACKNOWLEDGEMENTS}

We thank the EPSRC Grand Challenge in Nanotechnology for Healthcare (EP/G0620064/1), I-sense EPSRC IRC in Early Warning Sensing Systems for Infectious Diseases (EP/G062064/1), Royal Society (RS), Targanta Therapeutics, Bio Nano Consulting (BNC), the European Union FP7 Project VSMMART Nano (managed by BNC) for funding. The authors also thank J. Russat (London Centre for Nanotechnology), S. Sivachelvam (London Centre for Nanotechnology), M. Rehak (Sphere Fluidics, UK), R. A. Weiss (University College London) C. T. Verrips (QVQuality, Utrecht), T. Philips (Utrecht University), M. Morfini (University of Florence), T. Cass (Imperial College), V. Emery (Surrey Business School) and G. Aeppli (Paul Scherrer Institut) for the kind gift of materials and for helpful discussions. The glycoprotein antigens (gp140CN54 and gp140UG37) to llama antibody fragments were provided by the Centre for AIDS Reagents, National Institute for Biological Standards and Control (NIBSC) of the UK Medicines \& Healthcare Products Regulatoiry Agency (MHRA).

\section{METHODS}

Surface receptor coating procedure. A strategy of cantilever surface coating without Si underside passivation (see Supplementary Section 'case I') and with Si surface passivation (see Supplementary Section 'case III') as well as in-line referencing against control cantilevers were adopted to investigate how to improve the signal amplification, specificity, sensitivity and reproducibility of direct mechanical assays. To benchmark nanomechanical cantilever sensing to the surface plasmon resonance (SPR), we used plain $A u$ SPR sensor chips and with $\mathrm{N}$ Hydroxysuccinimide (NHS) thiolated surface linkers to couple receptors to the $\mathrm{Au}$ surface. The process was repeated for a covalently-attached carboxymethylated dextran (CM5) chip (see Supplementary Section 'case II' and 'case IV').

Cantilever measurements. A functionalised cantilever sensor array was mounted in a sealed liquid flow-cell with a volume of approximately $80 \mu \mathrm{l}$. The liquid cell and ligand solutions were placed into the temperature-controlled cabinet to undertake the experimental measurements. The absolute bending of the eight cantilevers was monitored using the Scentris (Veeco Instruments) optical beam device. Cantilevers were exposed to injections of different concentrations of Van and HIV antigens in sodium phosphate solutions at $\mathrm{pH} 7.4$ and at a constant ionic strength of $0.1 \mathrm{M}$ using a home-built gravity flow system and an automated pumping system (Model Genie Plus, Kent Scientific, Torrington, CT, USA) at optimised flow rate of $\sim 30-150 \mu \mathrm{lmin}^{-1}$. In the case of protein samples, the pumping system was used to control the flow rate of liquid samples in microfluidic channels at the flow rate of $\sim 30 \mu \mathrm{lmin}^{-1}$. All measurements of coagulation Factor (VIII) proteins were performed in distilled water as routinely used in the clinical applications. The raw data from three or four separate cantilever chips measured using a time-multiplexed optical detection system in each experiment was analysed to calculate the absolute, $\Delta \mathrm{z}_{\mathrm{abs}}$, bending deflections in $(\mathrm{nm})$ and subsequently converted into a differential surface stress signals, $\Delta \sigma_{\mathrm{abs}},\left(\mathrm{mNm}^{-1}\right)$ between the upper and lower sides of the cantilever according to the Stoney's equation (3): 


$$
\Delta \sigma_{a b s}=\frac{1}{3}\left(\frac{t}{L}\right)^{2} \frac{E}{1-v} \Delta z_{a b s}
$$

where $L$ is the effective length of the cantilever $\sim 500 \mu \mathrm{m}, t$ is the thickness $\sim 0.9 \mu \mathrm{m}$, $E /(1-v)=181 \mathrm{GPa}$ is the ratio between the Young's modulus $E$ and Poisson ratio $v$ of Si (100) and $\Delta z_{a b s}$ is the absolute cantilever bending deflection. The differential surface stress eq (VSR, coagulation Factor (VIII) and 2BF4) was calculated by subtracting in-situ reference $\Delta \sigma_{\mathrm{abs}}$ (PEG or LAB5) surface stress signal from the absolute stress signals, $\Delta \sigma_{\text {abs }}(\mathrm{VSR}$, coagulation Factor (VIII) and 2BF4)). In this report, a negative deflection signal corresponds to the downward bending of the cantilevers due to a compressive surface stress in which the cantilevers bend away from the surface receptors and a positive deflection corresponds to the upward bending of the cantilevers due to a tensile surface stress where the cantilevers bend toward the surface receptors.

SPR measurements and data analysis. Surface plasmon resonance or SPR arises from the conditions of total internal reflection of the conduction electrons, induced by the incident light at the interface between a metal and dielectric material (Supplementary Fig. 1). To establish the resonance conditions, the frequency of incident photons must match the natural frequency of surface electrons oscillating against the restoring force. The Biacore systems exploits SPR reflectivity measurements to monitor quantitatively the interactions between ligands and receptors at a surface ${ }^{31,32}$ in real time. Using SPR methodology, we performed assays using either single or multiple cycles and steady state measurements at $25^{\circ} \mathrm{C}$. Purified gp140 antigens and Van were diluted in HBS-EP buffer $(0.01 \mathrm{M}$ HEPES pH 7.4, $0.15 \mathrm{M} \mathrm{NaCl}, 3 \mathrm{mM}$ EDTA and 0,005\% P20) to quantify the affinity of ligand-receptor binding interactions. The diluted samples in phosphate buffer at $\mathrm{pH}$ 7.4 were injected in the flow cell for $2-5$ mins at a constant flow rate of $15 \mu \mathrm{min}^{-1}$ over the measuring and reference surfaces respectively. The bound ligand-receptor complexes were then allowed to dissociate for 1200 seconds. The kinetic constants (i.e. the rate for association constant, $k_{o n}$, and the dissociation rate constant, $k_{\text {off }}$ ) were computed from the binding curves using the BIAevaluation software and Eq. (2) to calculate the $K_{d}(\mathrm{Au})$ values.

The experimental error bars. The Surface-stress-data error bars and SPRresponse-data-error bars in each set of measurements were determined as the standard deviation of the surface stress data or SPR-response-data fitted from four separate chips of cantilever arrays chips and SPR chips respectively.

Competing surfaces reaction. To investigate whether the amplification of stress signal is controlled by the selective reactions at the two opposing cantilever top ( $\mathrm{Au})$ and bottom ( $\mathrm{Si}$ ) surfaces, we propose the hypothesis that 'the binding kinetics of a receptor molecule at each surface can act primarily as a sequencer of differential stress in response to surface coverage, where the reaction kinectics is decoupled'. To test this hypothesis, we implemented a model according to competing complex interactions between the two surfaces (Fig. 1a-c). This is a major advance because it means that probes can operate without the need for elaborate and lengthy 
passivation or sample labelling via surface manipulations applicable to all bilayered surface structures and should enable the realization of this technology for the detection of disease biomarkers present in extremely low concentrations.

To gain quantitative insights into the influence of receptor concentration on the mechanical response and to quantify the correlation between receptor distribution and stress generation, we determined the molecular footprint of mixed VSR and PEG at different concentrations using $\mathrm{X}$-ray photoelectron spectroscopy (XPS) (Supplementary Table S1 and Supplementary Table S2). By using this biologically relevant model experimental system, we uncover that even minor adjustments of surface receptor distribution has profound influence on the cantilever bending sensitivity (Fig. 4). In essence, from the chemical and conformational flexibility standpoint, adjusting the relative positions of receptor molecules, optimises the free energy of separation between them, whilst at the same time refines the accessibility of ligands to enhance the efficiency of surface stress generation. 
a)

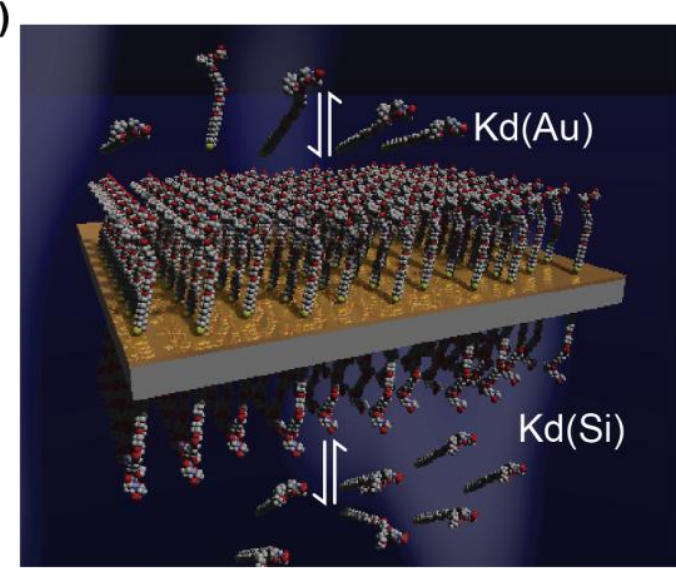

c)

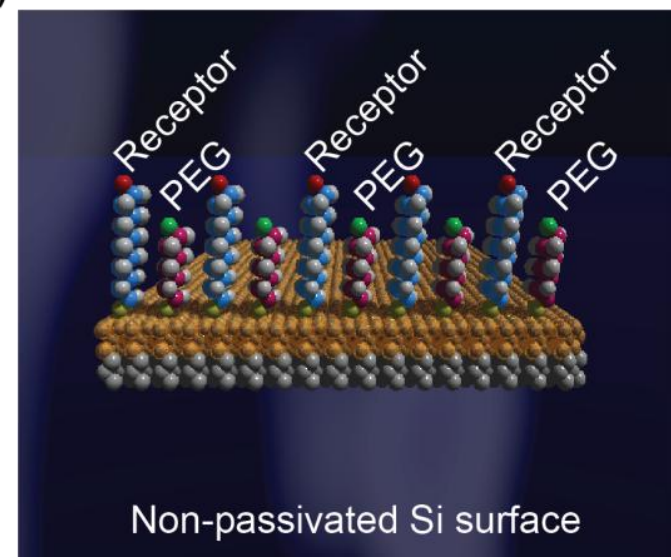

b)

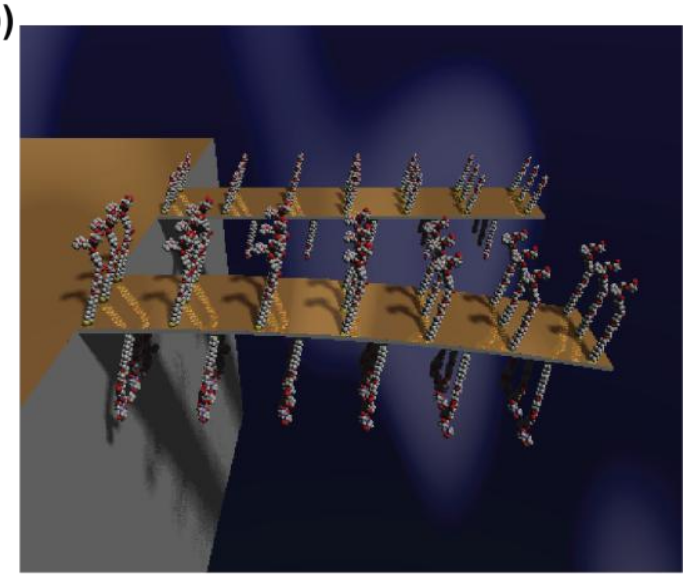

d)

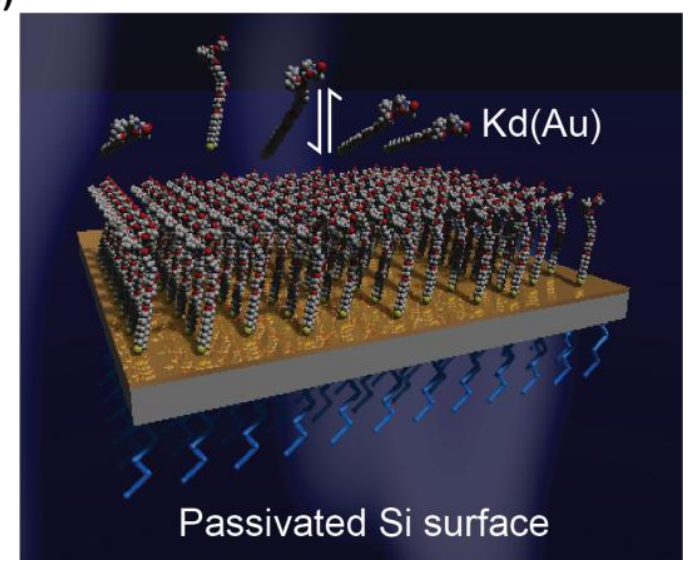

Figure 1: Nanomechanics of self-assembeled monolayer (SAM) to investigate the complex interplay between cantilever Au (top) and Si (bottom) surfaces against ligand-receptor binding interactions. a, Computer simulation image of solution and surface interactions, where the receptor molecules (grey vertical sticks with red headgroup circles) are immobilised on two surfaces ( $\mathrm{Au}$ and $\mathrm{Si}$ ) to form sensing layers. b, Schematic representation of rectangular Si cantilevers, measuring $500 \mu \mathrm{m}$ long, $100 \mu \mathrm{m}$ wide and $1 \mu \mathrm{m}$ thick, where the the receptor molecules (grey and red circles forming a vertical stick with red headgroup) are immobilised on the two surfaces ( $\mathrm{Au}$ and $\mathrm{Si}$ ) to form sensing layers. In $\mathbf{a}$ and $\mathbf{b}$, The efficacy of surface sensing layers was measured for a model bacteria cell wall precursors which present un-cross-linked peptide motifs terminating in the sequence Lysine-D-Alanine-DAlanine herein termed vancomycin susceptible receptor or VSR. c, Schematic representation showing a self-assembled monolayer (SAM) (grey and red circles forming a vertical stick with green headgroup), terminating in polyethylene glycol (PEG) incorporated with the surface receptors (grey and blue circles forming a vertical stick with red headgroup) in a well defined ratio to probe the effect of surface coverage on the efficiency of stress generation, where the Si surface was not passivated. d, Computer simulation image of solution and surface interactions, where the receptor molecules (grey and red circles forming a vertical stick with red headgroup) were immobilised at Au top surface only while the underlying Si surface was passivated using PEG-silane (vertical blue sticks) to prevent un-wanted reactions. In $\mathbf{a}$ and $\mathbf{d}$, The double arrows show the overall concept for the receptor immobilisation on the surface, where the receptor molecules (grey and blue circles forming a vertical stick with red headgroup) can be immobilised on a surface 
particularly the $\mathrm{Au}$ (top surface of cantilever) or Si (bottom surface of cantilever). $K_{d}$ $(\mathrm{Au})$ and $K_{d}(\mathrm{Si})$ are the equilibrium dissociation constants for $\mathrm{Au}$ and $\mathrm{Si}$ surfaces respectively. The cantilever arrays have the sensitivity to quantify competing binding interactions at the $\mathrm{Au}$ (top) and $\mathrm{Si}$ (bottom) surfaces in parallel and under identical conditions, with the surface reaction kinetics decoupled, and by tuning the receptor concentrations the impact of the underlying Si surface reactions can be minimized. 
a)

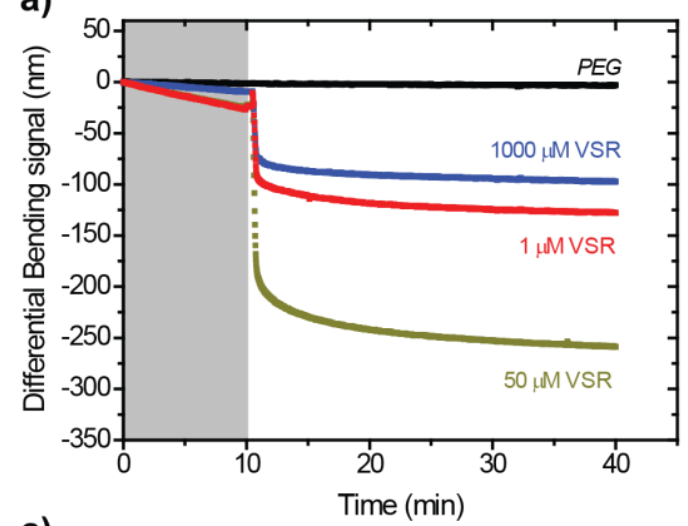

c)

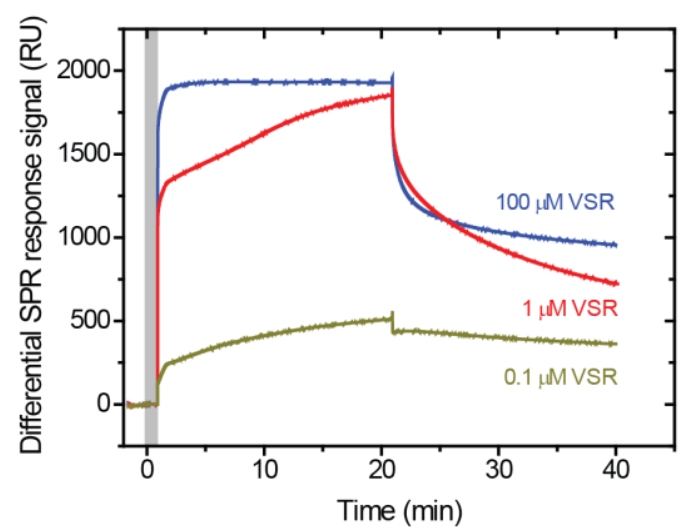

b)

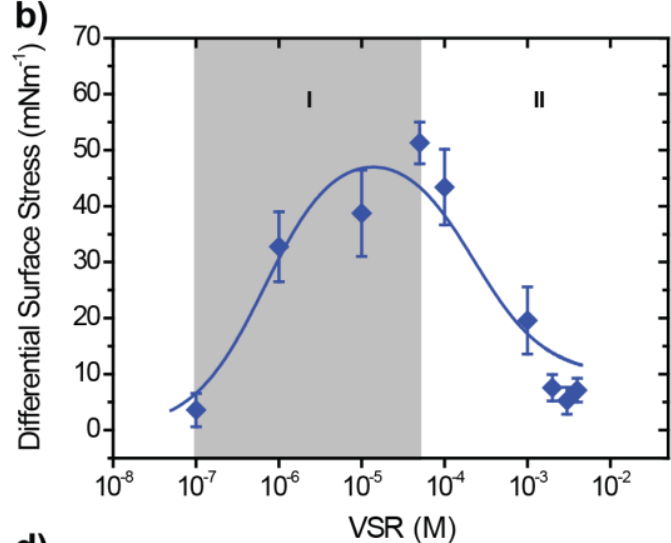

d)

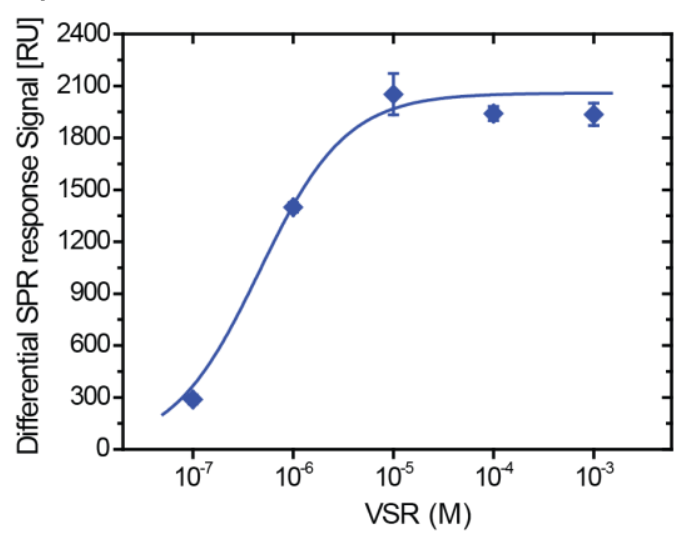

Figure 2: Nanomechanical and surface plasmon resonance (SPR) quantitation of surface binding reactions. a, The differential cantilever bending signal for $1 \mu \mathrm{M}$ (red), $50 \mu \mathrm{M} \mathrm{M}$ (dark yellow) and $1000 \mu \mathrm{M}$ (blue) of VSR against vancomycin fixed at $250 \mu \mathrm{M}$ to investigate the effect of surface chemistry on stress signaling. The differential PEG reference signal is shown in black. The cantilevers were found to bend downwards due to the steric and electrostatic repulsive interactions between bound ligand-receptor complexes. b, Semi-logarithmic plot showing measured differential surface stress response as a function of VSR concentration in solution against Van fixed at $250 \mu \mathrm{M}$ superimposed on the results of the fit according to Eq. (1) (solid lines) derived from model (I). c, The differential SPR response signals for $0.1 \mu \mathrm{M}$ (dark yellow), $1 \mu \mathrm{M}$ (red) and $100 \mu \mathrm{M}$ (blue) of VSR against vancomycin fixed at $250 \mu \mathrm{M}$ to investigate the effect of receptor concentration on signal amplification. In a-c, shaded areas represent the injection of sodium phosphate buffer without Van lasting for 10 minutes (cantilever measurements) or 2 minutes (SPR measurements) to establish a baseline and regime I of VSR concentration range where the differential surface stress signal was found to increase with increasing VSR concentration. d, Semi-logarithmic plot showing the measured differential SPR response signal as a function of VSR concentration in solution against vancomycin concentration fixed at $250 \mu \mathrm{M}$, superimposed on the results of the fit according to Eq. (2) (solid line) derived from model (II) to calculate $K_{d}(\mathrm{Au})$. In $\mathbf{b}$ and $\mathbf{d}$, The surfacestress-data error bars were determined as the standard deviation of surface-stressdata from four separate cantilever chips while the SPR-response-data-error bars were determined as the standard deviation of SPR-response-data fitted from four separate SPR chips. The measurements show that direct functionalisation of cantilevers without underside passivation can be achieved by the effective tuning of 
receptor concentrations in solution to minimise nonspecific reactions for sensitive and specific bioassays. 
a)

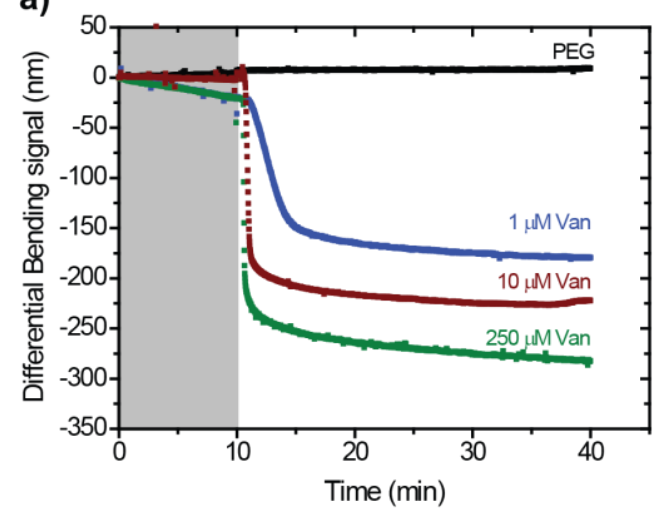

c)

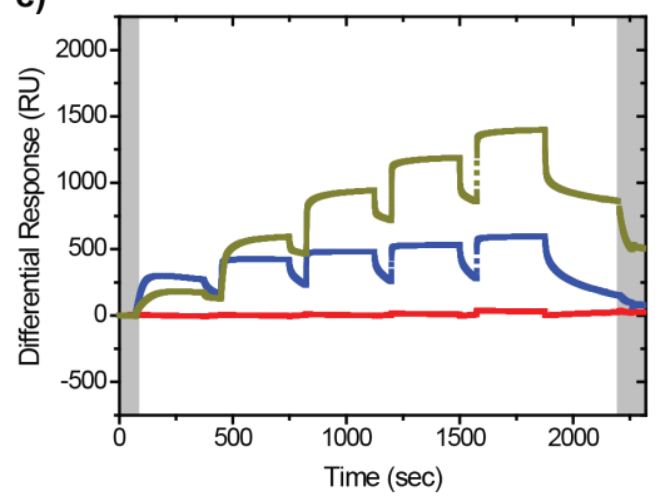

e)

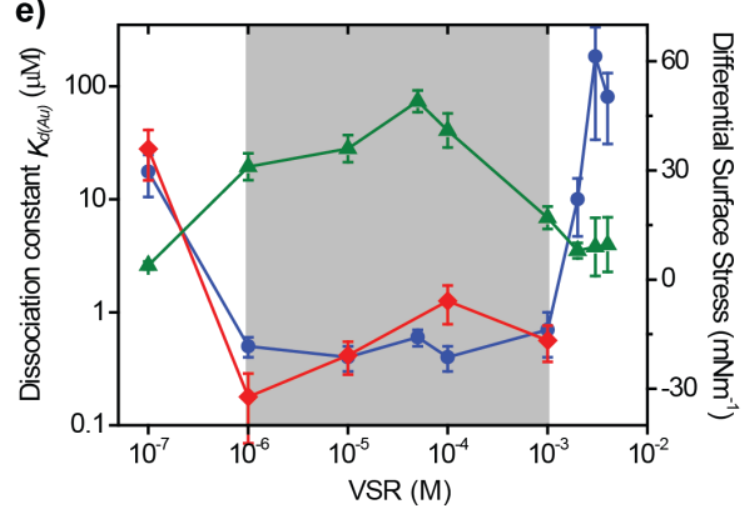

b)
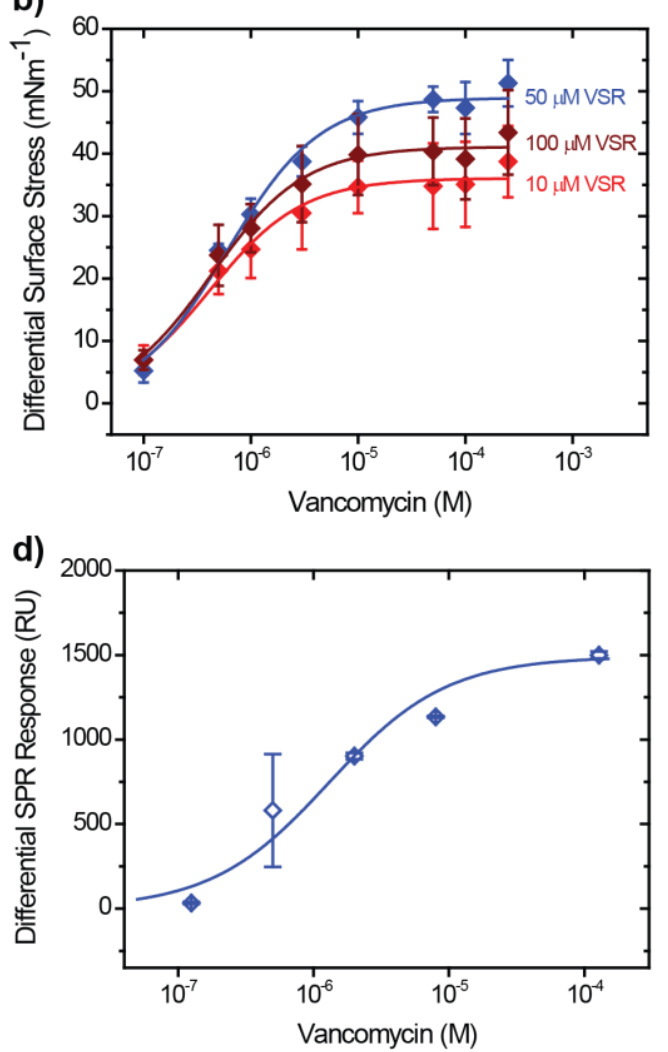

f)

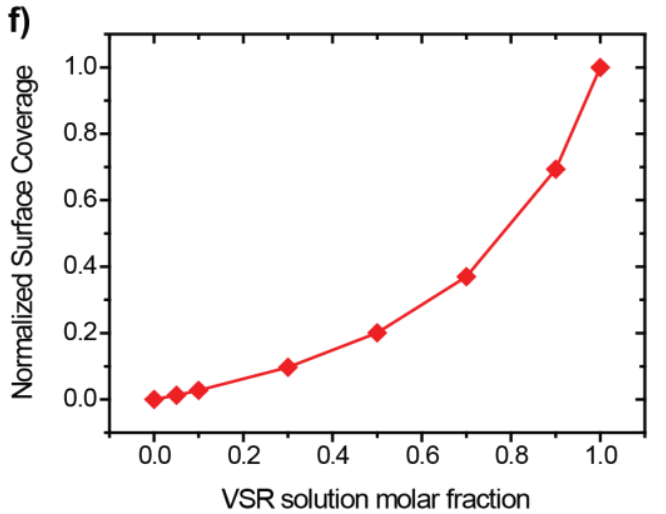

Figure 3: Quantitation of ligand-receptor interactions at fixed VSR concentrations using nanomechanical and surface plasmon resonance (SPR) assays. a, Differential bending signals at $50 \mu \mathrm{M}$ VSR against $1 \mu \mathrm{M}$ (blue), $10 \mu \mathrm{M}$ (wine) and $250 \mu \mathrm{M}$ (olive) of Van. The differential polyethylene glycol (PEG) reference signal is shown in black. A negative signal corresponds to a compressive surface stress which results in cantilever downward bending deflection. b, Semilogarithmic plot showing measured differential surface stress response for VSR fixed at a total solution concentration of $10 \mu \mathrm{M}$ (red), $50 \mu \mathrm{M}$ (blue) and $100 \mu \mathrm{M}$ (wine) against vancomycin concentration in solution, superimposed on the results to Eq. (2) (solid lines) derived from model (II) to calculate $K d(\mathrm{Au})$. c, The differential SPR response signals using unmodified Au-coated SPR sensor chips for $100 \mu \mathrm{M}$ VSR against $0.1 \mu \mathrm{M}$ (red), $1 \mu \mathrm{M}$ (blue) and $100 \mu \mathrm{M}$ (dark yellow) of Van. The differential sensorgram was obtained by subtracting PEG reference coated sensor signal from VSR signal. d, Semi-logarithmic plot showing the measured SPR differential response for surface bound receptors as a function of vancomycin concentrations in 
solution, superimposed on the results of the fit according to Model (II) Eq. (2) (blue solid line) for vancomycin (open blue diamonds) with the fitting parameters as $R U_{\max }$ $(\mathrm{Au}), n$, and $K_{d}(\mathrm{Au})$. e, Semi-logarithmic plot showing the measured differential surface stress response (green) and surface equilibrium dissociation constants, $K_{d}$ (Au) obtained by cantilevers (blue) and SPR (red) as a function of vancomycin exposed to different concentrations of VSR. In a, c and $\mathbf{e}$, The greyed-out area represents the injection of sodium phosphate buffer without Van lasting for 10 minutes (cantilever measurements) or 2 minutes (SPR measurements) to establish a baseline and the regime displaying a constant dissociation constant of antibiotic binding even when the surface stress signal is significantly different for the VSR concentrations in the range $1 \mu \mathrm{M}$ to $1000 \mu \mathrm{M}$. In $\mathbf{b}$, $\mathbf{d}$ and $\mathbf{e}$, The surface-stress-data error bars were determined as the standard deviation of surface-stress-data from four separate cantilever chips while the SPR-response-data-error bars were determined as the standard deviation of SPR-response-data fitted from four separate SPR chips. f, Plot showing the measured normalized surface coverage (solid red diamond squares) obtained by using X-ray photoelectron spectroscopy (XPS) as a function of the molar fraction of receptor (diluted with PEG in solution). In $\mathbf{e}$ and $\mathbf{f}$, The solid lines (green, blue and red) are not from thefits but instead as guide to the eye. These findings clearly show that the number of ligand-receptor interactions increases with coverage, but there is a threshold in the surface footprint required to generate a mechanical response. 
a)

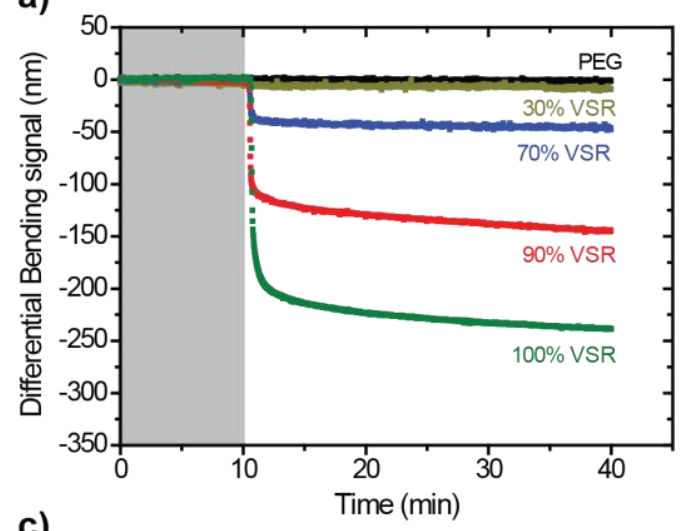

c)

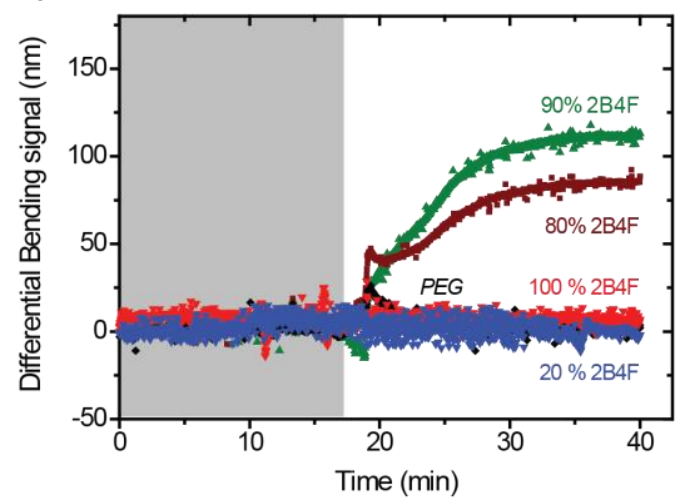

b)

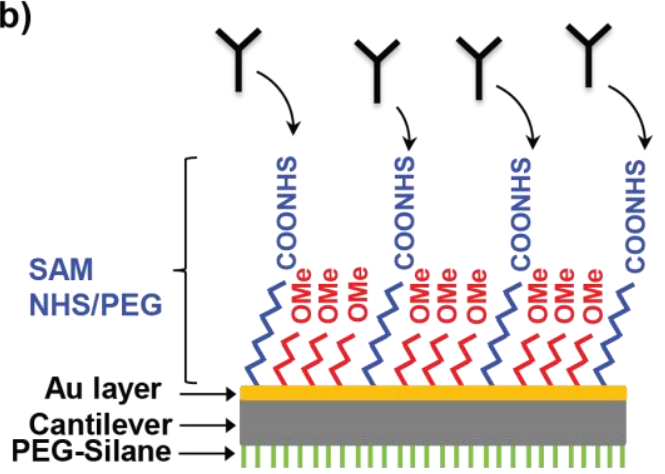

d)

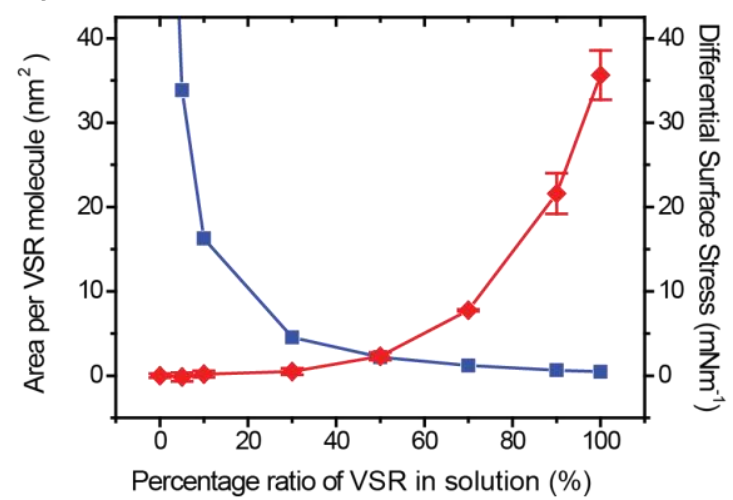

Figure 4: Investigating the effect of surface footprint (area per each receptor) on the ligand binding. a, The differential cantilever bending response signals in sodium phosphate buffer solution for a defined percentage ratio of $30 \%$ (dark yellow), $70 \%$ (blue), $90 \%$ (red), and $100 \%$ (olive) of VSR (diluted with PEG in solution) fixed at a total receptor solution concentration of $1 \mu \mathrm{M}$ at which the net cantilever stress signal contribution from the underlying $\mathrm{Si}$ reactions is negligible when exposed against vancomycin at $250 \mu \mathrm{M}$. A negative signal corresponds to a compressive surface stress which results in cantilever downward bending deflection. b, Schematic representation, in which the coupling approach introduces a receptor molecule into a thiol group anchored on the $\mathrm{Au}$ (top) surface by the modification of reactive $\mathrm{N}$ Hydroxysuccinimide (NHS) ester groups with primary amines, subsequently covalently linking a receptor molecule at a surface. Self-assembled monolayer (SAM) terminating in polyethylene glycol (PEG) or OMe in red was incorporated with receptors in a defined ratio to enhance biospecific binding efficiency of the sensing layer by reducing nonspecific binding on the $\mathrm{Au}$ surface $^{35}$. Passivation of the cantilever Si surface was achieved by using PEG-silane (vertical olive sticks) to block nonspecific underside reaction ${ }^{21}$. c, Differential response signals in sodium phosphate buffer for a defined percentage ratio of $20 \%$ (blue), $80 \%$ (wine), $90 \%$ (olive), and $100 \%$ (red) of llama-derived heavy chain variable domain antibodies $(\mathrm{VHH} \sim 15 \mathrm{kDa})^{36}$ herein termed 2B4F fixed at a total solution concentration of $2 \mathrm{mM}$ exposed to a constant human immunodeficiency virus type 1 (HIV-1) recombinant antigen derived from subtype A (gp140UG37) at $50 \mu \mathrm{M}$ gp140UG37. A positive signal corresponds to a tensile surface stress which results in cantilever upward bending deflections. In a and c, shaded areas represent the injection of sodium phosphate buffer without Van or gp140CN54 for control measurements lasting for 10 and 15 mins respectively to establish a baseline. d, Plot showing the differential 
surface stress response (red) to vancomycin at different concentrations and the area per VSR molecule (blue) as a function of the percentage ratio of VSR in solution (\%). The solid lines (blue and red) are not from fits but instead as a guide to the eye. The surface-stress-data error bars were determined as the standard deviation of surfacestress-data from four separate cantilever chips. The findings reveal that the efficiency of stress generation, in case of proteins (gp140UG37 140 kDa) is strongly linked to the surface molecular footprint. In contrast, for small molecules such as Van $(\sim 1.4$ $\mathrm{kDa}$ ), the stress is maximised when the receptor packing density is highest. 
a)

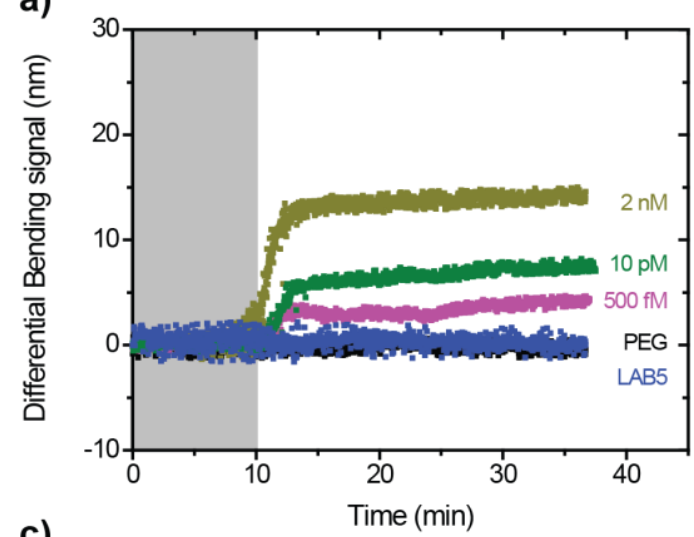

c)

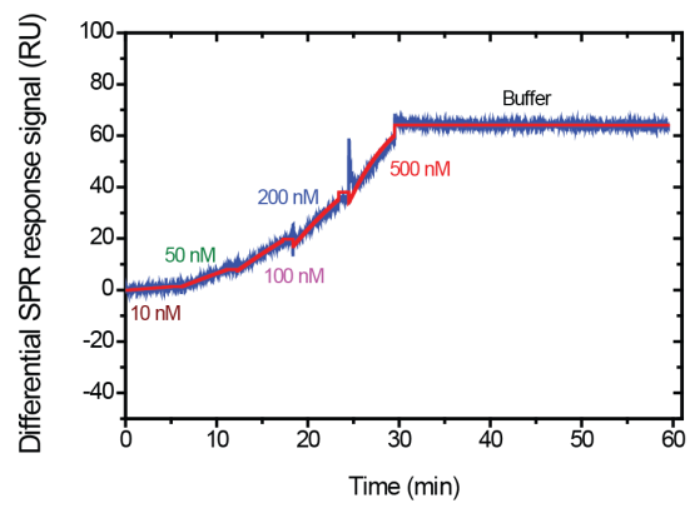

b)

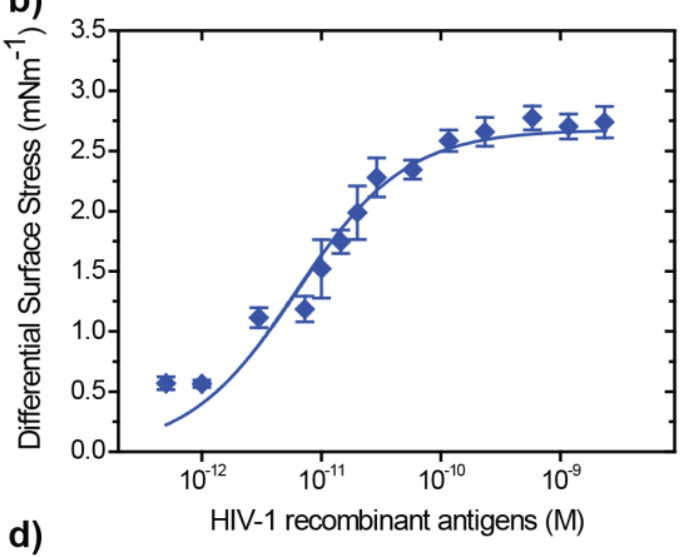

d)

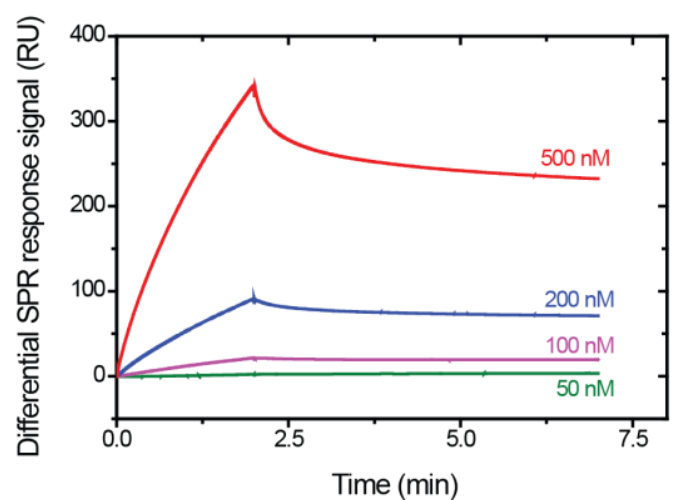

Figure 5: Comparative analysis of mechanical and surface plasmon resonance (SPR) biosensors of quantitative monitoring of protein interactions. a, The differential bending signals in buffer for llama-derived heavy chain fragment antibody $(\mathrm{VHH} 15 \mathrm{kDa})^{36}$ herein termed 2B4F against $2 \mathrm{nM}$ (dark yellow), $10 \mathrm{pM}$ (olive) and $500 \mathrm{fM}$ (mangenta) of HIV-1 recombinant antigens derived from B/C subtypes (gp140CN54). The differential polyethylene glycol (PEG) reference signal is shown in black. The comparative undetactable response of differential surface stress of nonspecific $\mathrm{VHH}$ protein, LAB5 ( 15 kDa) (blue) and PEG-coated cantilevers against gp140CN54 provides a strong evidence of surface-specific binding interactions between 2B4F and HIV-1 recombinant antigens. The greyed-out area represents the injection of sodium phosphate buffer without gp140CN54 for control measurements lasting for 10 mins to establish a baseline. b. Semi-logarithmic plot showing the measured differential surface stress response for surface bound receptors as a function of gp140CN54 concentrations in solution, superimposed on the results of the fit according to Model (II) Eq. (2) (solid line in blue) for gp140CN54 (solid symbols in blue) with the fitting parameters as $\sigma_{\max }, n$, and $K_{d}(\mathrm{Au})$. The surfacestress-data error bars were determined as the standard deviation of surface-stressdata from four separate cantilever chips. c, Differential response signals from a covalently-attached carboxymethylated dextran SPR sensor chips (CM5) single cycle kinectic measurements in sodium phosphate buffer solution for $10 \mathrm{nM}$ (wine), $50 \mathrm{nM}$ (olive), $100 \mathrm{nM}$ (magenta), $200 \mathrm{nM}$ (blue) and $500 \mathrm{nM}$ (red) gp140CN54. The differential sensorgram (solid line in blue) of gp140CN54 binding to 2B4F. The solid line (red) is the results of the fit computed from the binding curves using the BIAevaluation software using a two-state model superimposed on the measured differential response as a function of gp140CN54 concentrations in solution against the 2B4F. d, The differential SPR response signals using unmodified Au-coated SPR 
sensor chips for 2B4F against $50 \mathrm{nM}$ (olive), $100 \mathrm{nM}$ (magenta), $200 \mathrm{nM}$ (blue) and $500 \mathrm{nM}$ (red) of gp140CN54. In c,d, The differential response signal for gp140CN54 binding to 2B4F was obtained by subtracting the LAB5 reference or PEG coated sensor signals from 2B4F signals. The results demonstrate that direct mechanical detection sensitivity is superior. 

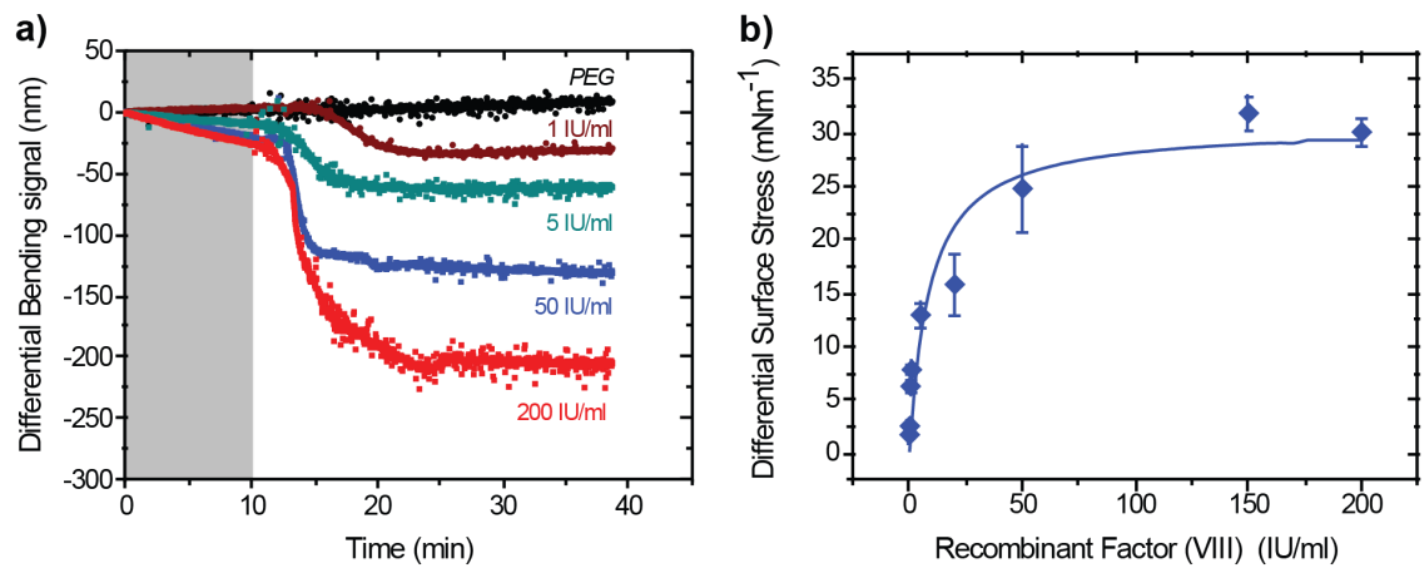

Figure 6: Nanomechanical quantitation of clotting factors for bleeding related disorders. a, The differential bending signals in distilled water for anti-Factor (VIII) against $1 \mathrm{IUml}^{-1}$ (wine), $5 \mathrm{IUml}^{-1}$ (olive), $50 \mathrm{IUml}^{-1}$ (blue) and $200 \mathrm{IUml}^{-1}$ (red) of Factor (VIII). The greyed-out area represents the injection of distilled water without Factor (VIII) for control measurements lasting for 10 mins to establish a baseline. A negative signal corresponds to a compressive surface stress which results in cantilever downward bending deflection. b. Plot showing the measured differential surface stress response for surface bound receptors as a function of Factor (VIII) concentrations in solution, superimposed on the results of the fit according to Model (II) Eq. (2) (solid line) for Factor (VIII) (solid symbols in blue) with the fitting parameters as $\sigma_{\max }, n$, and $K_{d}(\mathrm{Au})$. The surface-stress-data error bars were determined as the standard deviation of surface-stress-data from three separate cantilever chips. Here, we demonstrate that it is possible to use multiplexed nanomechanical cantilever sensors to quantitatively monitor clotting factors at clinically relevant concentrations and our work paves the way such that in the future, a suitably engineered surface probe such as miniaturised cantilever arrays could be paired with multiplexed Point-of-Care (PoC) devices for quantitative diagnoses of blood clotting disorders and anticoagulation therapy. 